\title{
Predictors for early motor improvement in patients with ischemic stroke
}

\author{
STANESCU Ioana C ${ }^{12}$, BULBOACA Angelo C. ${ }^{12}$, DOGARU Gabriela B. ${ }^{12}$, GUSETU Gabriel ${ }^{12}$,
} FODOR Dana M. ${ }^{1}$

Corresponding author: DOGARU Gabriela B.: $\underline{\text { dogarugabrielaumf@gmail.com }}$

\begin{abstract}
Disability as a stroke consequence is reported by $3 \%$ males and $2 \%$ females in general population. Motor deficits are common in stroke patients, but their complete recovery is seen only in a minority of cases. Assessment of motor deficits uses clinical methods, especially standardized scales, but also electrophysiological and imagistic methods. The motor recovery is a continuous process, maximal in the first month after stroke, decreasing gradually over the first 6 months. Most powerful predictors for motor recovery are clinical parameters: severity of motor deficit, onset of first voluntary movements after stroke in the first 48-72 hours, a continuous improvement in motor function during the first 8 weeks, a good postural control during the first month, young age, male sex, left hemispheric stroke and absence of other neurological impairments are strong positive predictors. Presence of motor-evoked potentials in paretic muscles and imagistic parameters as location, stroke volume and motor pathways integrity are paraclinical predictors for recovery. There are no specific biomarker which is efficient in predicting recovery. In patients with poor chances for recovery according to actual predictors, the development of more precise algorithms to assess functional outcome is needed, in order to support the choice of appropriate methods and intensity of rehabilitation treatment.
\end{abstract}

Key words: ischemic stroke rehabilitation, functional assessment, motor improvement, recovery predictors, prognostic factors,

\section{Introduction}

Stroke is an important cause of disability and mortality worldwide. Stroke prevalence is reported to be $2,9 \%$ in the US, according to recent statistics (1), ischemic strokes representing $87 \%$. Stroke incidence is lower in women and in young and middle age groups, but in older groups, stroke incidence in women is equal or even higher than in men.

Disability as a stroke consequence is reported by $3 \%$ males and $2 \%$ females in general population (1). The most common functional impairments after stroke are: motor deficits, language troubles, neglect, visual field deficits, sensory deficits, coordination troubles, cognitive dysfunctions and depression (2). Assessment of lost body functions should be based on objective methods: global scales, such as the NIHSS (National Institute of Health Stroke Scale), or specific scales for each domain, such as the motor section of Fugl-Meyer scale (3), gait velocity or Western Aphasia Battery, for example.

Recovery phases in stroke have been divided in hyperacute - the first $24 \mathrm{~h}$, acute - the first week, early subacute - from 1 week to 3 months, late subacute - between 3 to 6 months, and chronic - after 6 months post-stroke (4).

In neurologically stable patients, initial functional assessment should be performed in the first 24 hours after admission, by a multidisciplinary rehabilitation team (2). Assessment of functional abilities (measured by the Barthel Index, modified Rankin Scale and Functional Independence Measure FIM), activity limitations (measured by ADLs and IADLs) and participation restrictions are also important (2). Motor deficits in strokes are common, being caused by lesions of the motor areas and pyramidal tract. Intensity of motor deficit is related to stroke location and lesion area. Unfortunately, in many cases, the motor deficit in upper limb is severe: 30 to $66 \%$ of patients will not recover upper limb motor function at 6 months post-stroke, $25 \%$ will show partial improvement and only 5 to $20 \%$ will improve completely (5). In strokes located in the middle cerebral artery (MCA) territory with complete 
hemiplegia at onset, $62 \%$ of patients showed no improvement at 6 months (5). The inability to use the upper extremity is directly related to autonomy loss (inability to work, to drive, to assure self-care) and disability (2). Lower limb complete motor deficit is present in $20 \%$ of acute and subacute stroke patients; among them, $21-40 \%$ will remain with severe plegia, $23-34 \%$ will improve and only $10 \%$ will regain walking (6).

Despite advances in rehabilitation methods, patients achieved $63 \%$ of proportional recovery potential at 3 months and $78 \%$ at 6 months, proving the limits of biological repair processes and of rehabilitation treatments (7).

Assessment of motor deficits. Includes clinical, electrophysiological and imagistic methods. Clinical assessment include: active movements, muscle strength, muscle tone, balance, coordination (as assessed by the standard neurologic examination), but also finger and upper limb function, lower limb function and gait. Functional scores, such as National Institutes of Health Stroke Scale (NIHSS) score and modified Rankin Scale (mRS) calculated at admission are basic tools in outcome assessment (8). Other specific scales are used to quantify the intensity of motor deficit in stroke rehabilitation: the Fugl-Meyer Test (FMA) and the Action Research Arm Test (ARAT) $(3,5,9,10)$. For an accurate assessment of real-life impairments in outpatients, standardized questionnaires measuring patient selfreported performances and individual electronic devices (pedometers, ankle- or wrist-accelerometers, step-monitors) are useful (2).

Electrophysiological studies are useful to assess the intensity and evolution of post-stroke motor deficit; mostly used is transcranial magnetic stimulation (TMS) with recordings of motor evoked potential (MEP) in different muscle groups (11).

Neuroimagistic studies are also important for predicting motor recovery, because they assess lesion location (MRI with diffusion-weighted imaging DWI sequences), damage of motor pathways (diffusion tensor imaging DTI sequences) and patterns of cortical networks activation during rehabilitation procedures (functional MRI - fMRI) (12).

Physiopathology of motor recovery. Is a process based on neuroplasticity. Lesions of the motor cortex and / or pyramidal tract lead to adaptative changes in the surrounding cortical areas with new neural networks formation. This neural plasticity process is involved in the recovery of the lost motor function after stroke. Reconstruction of motor networks and pathways requires specific cellular and molecular processes which are intensively studied. Recovery process is spontaneous, but is also facilitated by motor learning during rehabilitation program. Compensation is a synergic process, meaning that a task which cannot be accomplished by the damaged brain area is instead performed by another brain area which uses new motor patterns form the same result (13).

Biological markers for recovery. Biomarkers of stroke recovery are indicators of ongoing processes at cellular or molecular levels, which are used to predict and monitor rehabilitation outcome (14). The biomarkers that have been studied in stroke rehabilitation are $\mathrm{S} 100$ calcium binding protein $\mathrm{B}, \mathrm{C}$ reactive protein (CRP), matrix metalloproteinases (MMPs), N-terminal pro-brain natriuretic peptide (NT-pro-BNP), interleukin-6 (IL-6) $(15,16)$. Levels of NT-pro-BNP are correlated with NIHSS score values and are usefull in predicting prognosis in patients with acute ischemic stroke, but have no value in predicting motor recovery $(17,18)$. Also, IL6 did not shown to have predictive value in stroke outcome (47). Matrix metalloproteinases (MMPs) are enzymes involved in remodeling of the extracellular matrix, their expression is correlated with tissular injury, apoptosis, but also with tissue repair and remodeling after stroke $(19,20)$. The hypothesis that MMPs might be used as biomarkers of treatment efficacy in stroke rehabilitation is not proved yet. Plasmatic MMP 12 and 13 high levels are observed in severe strokes with poor outcome, but MMP3 level is increased in patients with better outcome and substantial recovery after stroke (19). Recent studies have identified drugs which can modulate MMPs expression and influence oxidative stress parameters, such as curcumin $(20,21)$ but their value in stroke recovery is not demonstrated. Other studies have demonstrated that in patients in subacute recovery phase, regular kinesitherapy will improve plasmatic antioxidant capacity, but not oxidative stress parameters (22). Another study demonstrates that levels of antioxidant enzyme $\mathrm{Cu} / \mathrm{Zn}$ Superoxide Dismutase $(\mathrm{Cu} / \mathrm{Zn}$ SOD) are lower in stroke patients than in controls, but increase after intensive neurorehabilitation. Thus, $\mathrm{Cu} / \mathrm{Zn}$ SOD levels could 
be used as a positive predictive biomarker of recovery and treatment efficacy (23).

Clinical predictive factors for motor recovery. Motor deficits are common in stroke patients, but their complete recovery is seen only in a minority of cases. The most powerful predictors for motor recovery are the clinical parameters.

Young age, male sex and left hemispheric stroke are predictors for better motor recovery (24), but the most important clinical predictor is the severity of motor deficit in the early days after stroke: patients with moderate or mild motor deficits have better prognosis in functional recovery than patients with severe hemiplegia $(24,25)$. This statement is also reflected by NIHSS score: a score of less than 6 points in the first 24 hours indicates a good recovery at 3 months, but a score of more than 16 points indicates a poor outcome with important disability (26).

Motor improvement in upper limb can be monitored by weekly assessments of finger extension and shoulder abduction movements, in the first 8 weeks after stroke (25). Assessment of shoulder-abduction and finger-extension movements have been included in a model - SAFE model- which predict with accuracy upper limb recovery in stroke patients (27). Full recovery of upper limb function is obtained by $60 \%$ of the patients who performed some degree of finger extension at 72 hours after stroke (28). In patients which initially were unable to perform voluntary finger extension at 72 hours after stroke, motor recovery of upper limb function is still possible and is predicted by spontaneous return of voluntary finger extension between 4 to 8 weeks post-stroke (25).

The onset of first voluntary movements in paralyzed limbs is another important predictor of motor improvement. The motor recovery is a continuous process, which is maximal in the first month after stroke onset, then decreases gradually over the first 6 months (13). In the first 72 hours after stroke, presence of voluntary movements in the affected upper limb, especially finger extension and shoulder abduction, is considered to be a good predictor of regaining upper limb function with $98 \%$ probability of improvement at 6 months (28). If voluntary movements appeared at the shoulder, fingers and lower limb in the first 7 days after stroke, it reflects a strong probability of improvement in upper limb motility and gait (12). Even in patients without voluntary upper limb movements, the occurrence after the first week of active movements in the affected lower limb predicts $74 \%$ chances to regain upper limb motility at 6 months. Contrarily, patients with plegic upper and lower limb at 1 week have only $14 \%$ chances predicted to recover their upper limb function (5). At two weeks after stroke, another study showed that patients with persistent upper and lower limb complete motor deficit had poor outcome for motor recovery at 6 months (29). If grip movements are absent at 1 month, the prognosis for recovery is poor. Another study has narrowed the time window for motor recovery, showing that maximal improvement of upper limb function is obtained in 3 weeks by $80 \%$ of patients, and in 9 weeks by $95 \%$ of patients (30).

Another strong predictor of motor recovery is the improvement rate of motor deficit in the first month post-stroke. A continuous improvement in motor function at 2 months after stroke, will increase the chances for 6 months recovery for these patients. Kwakkel et al. have shown that $94 \%$ of patients in which the motor score at Fugl-Meyer scale improves to more than 19 points in the first month, have better recovery chances than patients with scores below that limit, which have only $9 \%$ chances to achieve some degree of active motility (5).

Recovery of lower limb motility is more difficult to assess; it have been shown to be predicted by stroke severity and lesion volume (6). A variable pattern of recovery is demonstrated in patients with severe lower limb plegia. Patients which achieve good control of their trunk posture during the first week after stroke, can perform more intense physical activity in the first year post-stroke, despite comorbidities, indicating a better recovery $(31,32)$. Also, the patients which are capable to "out-of-bed" mobilization early after stroke, could achieve a better outcome, but this parameter could in fact be related to a milder severity of stroke (33). The measure of walking speed in patients who can walk remains a useful tool for assessing gait ability (2).

A negative predictor is the association of other concomitant neurological deficits, such as visuospatial neglect, somatosensory dysfunctions and cognitive troubles, which will decrease the probability of functional improvement of motor deficits $(24,25)$. In those patients, the motor recovery process is delayed; however, neglect is known to improve in the first 3 months, giving better chances for motor improvement after that interval. 
Paraclinical predictors for motor function recovery are also important. Presence of motor and sensory evoked potentials at TMS is associated with a favorable outcome in upper limb rehabilitation (24). Precisely, the presence of motor-evoked potential (MEP) in abductor digiti minimi 7 days after stroke, is a positive predictor for hand dexterity (11). At 3 weeks post-stroke, the registered MEP has the same predictive value as clinical assessment of upper limb FMA score (11). However, if MEP can be elicited by TMS in affected upper limb muscles within first days after stroke, a good recovery prognosis is predicted (34). In the lower limb, presence of a MEP indicates higher chances in having gait autonomy at 1 year (35). TMS with MEP measurement can be used to predict recovery even in chronic stages, when the relationship $\mathrm{MEP}+$ and improvement is preserved (14).

One electroencephalography (EEG) parameter (e.g. coherence in beta frequency band between the motor cortex on the lesional site and the rest of the cortex) could be used as predictor for motor recovery at 3 months (36).

MRI parameters related to stroke location, volume and motor pathways integrity were used to predict recovery. Concerning functional recovery measured by the ability to perform ADL (activities of daily living), assessment of MRI parameters did not change the prognosis predicted by clinical variables (37). However, MRI gives precise structural details, and is used by many studies to assess the impact of lesion location on stroke recovery. Lesions located in corona radiata, insula, opercular cortex, right inferior parietal lobe, left superior and middle temporal cortex predicts an unfavorable motor recovery in moderate to severe strokes in the middle cerebral artery (MCA) territory (34). The concomitant presence of diffuse white matter disease, known as leukoaraiosis, is associated with more severe strokes (14).

Also, a decrease in ADC (apparent diffusion coefficient) signal in the ipsilateral cerebellar peduncle and posterior limb of internal capsule predicts poor recovery (12). For patients with severe motor deficit, measurement of pyramidal tract fiber number by diffusion tensor imaging (DTI), could predict motor recovery assessed by Fugl-Meyer score (38). Higher fractional anisotropy (FA) of the pyramidal tract on the lesional and contralateral site in early phases is correlated with a better recovery (39). Other authors have calculated the pyramidal tract lesion load, and demonstrated that it have a predictive value for motor recovery at 3 months (40). Resting state functional connectivity (rsFC) between primary motor cortex and other cortical areas is decreased in patients with severe motor deficits at 6 months (41). A functional MRI (fMRI) parameter the laterality index - in primary motor cortex, could be a predictor for motor recovery, as it shows more diffuse pattern of activation in patients with poor outcome (14).

Algorithms for motor recovery. Clinical and paraclinical parameters were used together to design different algorithms for motor recovery. In the first 72 hours post-stroke, clinical parameters are most valuable: presence of active finger-extension and shoulder abduction could predict good recovery of upper limb function. After that, especially in patients with severe motor deficit, paraclinical methods - such as TMS, MRI, fMRI - are usefull, together with periodical clinical assessments, in detecting patients which still could have recovery potential and who could benefit of maximal rehabilitation treatment (34).

Variability of the recovery process could be related also to individual genetic factors, such as polymorphisms of the gene for brain-derived neurotrophic factor (BDNF), for example, which are influencing neuroplasticity and motor learning processes (12).

Conclusion. Identifying accurate prognostic factors for motor recovery in stroke is important for the patient and caregivers, who need information about expected outcome, but also for the rehabilitation team, in the selection of appropriate treatment modalities. For patients with severe motor deficit, in which clinical and paraclinical predictors indicate minimal recovery, the choice of rehabilitation strategies should focus on compensatory strategies. Nevertheless, for these patients, new therapeutic strategies which aim to stimulate spontaneous recovery are needed.

Future directions. Stroke research should focus on the development of early rehabilitation methods, which are able to induce maximal recovery in patients with severe motor deficits. These methods combine classical neurorehabilitation with brain stimulation techniques TMS or robotics-based rehabilitation $(42,43)$. In subacute stroke, studies 
have shown that intensive physical therapy in patients with severe motor deficit could improve recovery and walking (44). In the chronic phase of stroke, mild improvements in motor function are observed with compensatory strategies, and important improvements in quality of life are expected if rehabilitation program is continued in a balnear resort $(45,46)$.

\section{References}

1. Benjamin EJ, Muntner P, Alonso A, Bittencourt MS, Callaway CW, Carson AP, Chamberlain AM, Chang AR, Cheng S, Das SR, Delling FN, Djousse L, Elkind MSV, Ferguson JF, Fornage M, Jordan LC, Khan SS, Kissela BM, Knutson KL, Kwan TW, Lackland DT, Lewis TT, Lichtman JH, Longenecker CT, Loop MS, Lutsey PL, Martin SS, Matsushita K, Moran AE, Mussolino ME, O'Flaherty M, Pandey A, Perak AM, Rosamond WD, Roth GA, Sampson UKA, Satou GM, Schroeder EB, Shah SH, Spartano NL, Stokes A, Tirschwell DL, Tsao CW, Turakhia MP, VanWagner LB, Wilkins JT, Wong SS, Virani SS; American Heart Association Council on Epidemiology and Prevention Statistics Committee and Stroke Statistics Subcommittee. Heart Disease and Stroke Statistics-2019 Update: A Report From the American Heart Association. Circulation. 2019 Mar 5;139(10):e56-e528.

2. Winstein CJ, Stein J, Arena R, Bates B, Cherney LR, Cramer SC, Deruyter F, Eng JJ, Fisher B, Harvey RL, Lang CE, MacKay-Lyons M, Ottenbacher KJ, Pugh S, Reeves MJ, Richards LG, Stiers W, Zorowitz RD. Guidelines for Adult Stroke Rehabilitation and Recovery: A Guideline for Healthcare Professionals From the American Heart Association/American Stroke Association. Stroke. 2016;47:e98-e169.

3. Fugl-Meyer AR, Jääskö L, Leyman I, Olsson S, Steglind S (1975) The post-stroke hemiplegic patient. 1. a method for evaluation of physical performance. Scand J Rehabil Med. 7: 13-31 .

4. Bernhardt J, Hayward KS, Kwakkel G, et al. Agreed definitions and a shared vision for new standards in stroke recovery research: the Stroke recovery and rehabilitation roundtable taskforce. Int J Stroke. 2017; 12: 444-450.
5. Kwakkel G, Kollen BJ, Van der Grond J, Prevo AJH. Probability of regaining dexterity in the flaccid upper limb. Impact of severity of paresis and time since onset in acute stroke. Stroke. 2003;34:2181-2186.

6. Huang SL, Chen BB, Hsueh IP, Jeng JS, Koh CL, Hsieh CL. Prediction of lower extremity motor recovery in persons with severe lower extremity paresis after stroke. Brain Inj.2018;32(5):627-633 .

7. Stinear CM, Byblow WD, Ackerley SJ, Smith MC, Borges VM, Barber PA. Proportional motor recovery after stroke: implications for trial design. Stroke 2017; 48: 795-98.

8. Stroke unit trialists' collaboration: Organised inpatient (stroke unit) care for stroke. Cochrane Database of Syst Rev. 2013;11.

9. Lyle RCA. Performance test for assessment of upper limb function in physical rehabilitated treatment and research. Int J Rehabil. 1981; 4: 483-492.

10. Santisteban L, Térémetz M, Bleton JP, Baron JC, Maier MA, Lindberg PG. Upper Limb Outcome Measures Used in Stroke Rehabilitation Studies: A Systematic Literature Review. PLoS One. 2016;11(5):e0154792.

11. van Kuijk A, Pasman JW, Hendricks HT, Zwarts MJ, Geurts ACH. Predicting Hand Motor Recovery in Severe Stroke: The Role of Motor Evoked Potentials in Relation to Early Clinical Assessment. Neurorehabilitation and Neural Repair. 2009; 23(1): 45-51

12. Stinear C. Prediction of recovery of motor function after stroke. Lancet Neurol. 2010; 9: 1228-32.

13. Li S. Spasticity, Motor Recovery, and Neural Plasticity after Stroke. Front Neurol. 2017;8:120.

14. Boyd LA, Hayward KS, Ward NS, Stinear CM, Rosso C, Fisher RJ, Carter AR, Leff AP, Copland DA, Carey LM, Cohen LG, Basso DM, Maguire JM, Cremer SC.Biomarkers of stroke recovery: Consensus-based core recommendations from the Stroke Recovery and Rehabilitation Roundtable. Int J Stroke2017 Jul;12 (5):480-493. doi: $10.1177 / 174749301771417$.

15. Branco JP, Costa JS, Sargento-Freitas J, Oliveira S, Mendes B, Laíns J, Pinheiro J. Neuroimaging and Blood Biomarkers in Functional Prognosis after Stroke. Acta Med Port. 2016 Nov;29(11):749-754 . 
16. Whiteley W, Wardlaw J, Dennis M, Lowe G, Rumley A, Sattar N, Welsh P, Green A, Andrews M, Sandercock P. The use of blood biomarkers to predict poor outcome after acute transient ischemic attack or ischemic stroke. Stroke. 2012 Jan; 43(1):86-91.

17. Chen X, Zhan X, Chen M, Lei H, Wang Y, Wei $\mathrm{D}$, Jiang $\mathrm{X}$. The prognostic value of combined NT-pro-BNP levels and NIHSS scores in patients with acute ischemic stroke. Intern Med. 2012; 51(20):2887-92. Epub 2012 Oct 15 .

18. Stanescu IC, Dogaru G. Brain natriuretic peptide (BNP): biomarker for risk stratification and functional recovery prediction in ischemic stroke. Balneo Research Journal. 2015; 6(1):4044 .

19. Ma F, Rodriguez S, Buxo X, Morancho A, RibaLlena I, Carrera A, Bustamante A, Giralt D, Montaner J, Martinez C, Bori I, Rosell A. Plasma Matrix Metalloproteinases in Patients With Stroke During Intensive Rehabilitation Therapy. Arch Phys Med Rehabil. 2016 Nov;97(11):1832-1840. doi: 10.1016/j.apmr.2016.06.007. Epub 2016 Jul 1

20. Bulboacă AE, Porfire AS, Tefas LR, Boarescu PM, Bolboacă SD, Stănescu IC, Bulboacă AC, Dogaru G. Liposomal Curcumin is Better than Curcumin to Alleviate Complications in Experimental Diabetic Mellitus. Molecules.2019 Feb 27;24(5). pii: E846. doi: 10.3390/molecules24050846.

21. Bulboacă AE, Bolboacă SD, Stănescu IC, Sfrângeu CA, Bulboacă AC. Preemptive Analgesic and Antioxidative Effect of Curcumin for Experimental Migraine. Biomed Res Int.2017;2017:4754701. doi: 10.1155/2017/4754701. Epub 2017 Oct 24

22. Kihoin N, Tanaka K, Okuno M, Okamoto T, Saura R. Exercise attenuates oxidative stress in patients with stroke. Neurology Asia .2016; 21(1): $7-16$.

23. Ciancarelli I, Di Massimo C , De Amicis D, Pistarini C, Tozzi Ciancarelli MG. Uric Acid and $\mathrm{Cu} / \mathrm{Zn}$ Superoxide Dismutase: Potential Strategies and Biomarkers in Functional Recovery of Post-Acute Ischemic Stroke Patients after Intensive Neurorehabilitation. Current Neurovascular Research. 2015, 12, 120-127.

24. Coupar F, Pollock A, Rowe P, Weir C, Langhorne P. Predictors of upper limb recovery after stroke: a systematic review and meta-analysis.

Clin

Rehabil. 2012.

Apr;26(4):291-313 .

25. Winters C, Kwakkel G, Nijland R, van Wegen E; EXPLICIT-stroke consortium. When Does Return of Voluntary Finger Extension Occur Post-Stroke? A Prospective Cohort Study. PLoS One. 2016;11(8):e0160528.

26. Adams HP, Jr, Davis PH, Leira EC, et al. Baseline NIH Stroke Scale score strongly predicts outcome after stroke: a report of the Trial of Org 10172 in Acute Stroke Treatment (TOAST). Neurology.1999;53:126-31.

27. Wolf SL, Kwakkel G, Bayley M, McDonnell $\mathrm{MN}$, for the Upper Extremity Stroke Algorithm Working Group. Best practice for arm recovery post stroke: an international application. Physiotherapy

(2015),http://dx.doi.org/10.1016/j.physio.2015.0 8.007 .

28. Nijland RH, van Wegen EE, Harmeling-van der Wel BC, Kwakkel G; . Presence of finger extension and shoulder abduction within 72 hours after stroke predicts functional recovery: early prediction of functional outcome after stroke: the EPOS cohort study. Stroke. 2010 Apr;41(4):745-50.

29. Shelton F, Reding MJ. Effect of lesion location on upper limb motor recovery after stroke. Stroke. 2001;32:107-112.

30. Nakayama H, Jørgensen HS, Raaschou HO, Olsen TS. Recovery of upper extremity function in stroke patients: the Copenhagen Stroke Study. Arch Phys Med Rehabil. 1994 Apr;75(4):394-8.

31. Persson CU, Hansson PO, Lappas G, Danielsson A. Physical Activity Levels and Their Associations With Postural Control in the First Year After Stroke. Phys Ther. 2016 Mar 24.

32. Frîngu F, Guşetu G, Iosip A, Gurzău D, Dogaru G, Zdrenghea D, Pop D. The predictors of exercise capacity impairment in diabetic patients. Balneo Research Journal. 2017;8(2):26-32.

33. Franceschini M, Fugazzaro S, Agosti M, Sola C, Di Carlo A, Cecconi L, Ferro S, Italian Study Group on Implementation of Stroke Care (ISC Study). Acute Phase Predictors of 6-Month Functional Outcome in Italian Stroke Patients Eligible for In-Hospital Rehabilitation. Am J Phys Med Rehabil. 2018 Jul; 97(7):467-475 .

34. Stinear CM, Barber AP, Petoe M, Anwar S, Byblow WD. The PREP algorithm predicts 
potential for upper limb recovery after stroke. Brain. 2012: 135; 2527-2535.

35. Chang MC, Do KH, Chun MH. Prediction of lower limb motor outcomes based on transcranial magnetic stimulation findings in patients with an infarct of the anterior cerebral artery. Somatosens Motor Res .2015; 32: 249-253 .

36. Nicolo P, Rizk S, Magnin C, Pietro MD, Schnider A, Guggisberg AG. Coherent neural oscillations predict future motor and language improvement after stroke. Brain. 2015; 138: 3048-60.

37. Schiemanck SK, Kwakkel G, Post MW, Kappelle LJ, Prevo AJ. Predicting long-term independency in activities of daily living after middle cerebral artery stroke: does information from MRI have added predictive value compared with clinical information? Stroke. 2006 Apr;37(4):1050-4.

38. Bigourdan A, Munsch F, Coupe P, et al. Early fiber number ratio is a surrogate of corticospinal tract integrity and predicts motor recovery after stroke. Stroke .2016; 47: 1053-1059.

39. Wen H, Alshikho MJ, Wang Y, et al. Correlation of Fractional anisotropy with motor recovery in patients with stroke after postacute rehabilitation. Arch Phys Med Rehab. 2016; 97: 1487-1495.

40. Feng W, Wang J, Chhatbar PY, et al. Corticospinal tract lesion load: an imaging biomarker for stroke motor outcomes. Ann Neurol .2015; 78: 860-870.

41. Carter AR, Astafiev SV, Lang CE, et al. Resting interhemispheric functional magnetic resonance imaging connectivity predicts performance after stroke. Ann Neurol .2010; 67: 365-375.

42. Zarahn E, Alon L, Ryan SL, Lazar RM, Vry MS, Weiller C, Marshall RS, Krakauer JW. Prediction of motor recovery using initial impairment and fMRI $48 \mathrm{~h}$ poststroke. Cereb Cortex. 2011;21(12):2712-21.

43. Bulboacă AE, Bolboacă SD, Bulboacă AC.Ethical considerations in providing an upper limb exoskeleton device for stroke patients. Med Hypotheses. 2017 Apr;101:61-64. doi: 10.1016/j.mehy.2017.02.016. Epub .2017 Mar 2.

44. Kawakami K, Tanino G, Tomida K, et al. Influence of increased amount of exercise on improvements in walking ability of convalescent patients with post-stroke hemiplegia. J Phys Ther Sci. 2016 Jan. 28 (2):602-6.

45. Dogaru G, Ispas A, Bulboacă A, Motricală M, Stănescu I. Influence of balnear therapy at Băile Tuşnad on quality of life of post-stroke patients. Balneo Research Journal. 2017;8(4):201-205 .

46. Dogaru G, Ispas A, Stanescu I. A clinical study on the efficacy of natural therapeutic factors in Baile Tusnad for the rehabilitation of post-stroke patients. Balneo Research Journal. 2017;8(1):510.

47. Cheng B, Forkert ND, Zavaglia M, Hilgetag CC, Golsari A, Siemonsen S, et al. Influence of stroke infarct location on functional outcome measured by the modified rankin scale. Stroke. 2014; 45:1695-1702. 